\title{
Inverse Sequences and Absolute Co-Extensors
}

\author{
by \\ Ivan IVANŠIĆ and Leonard R. RUBIN
}

Presented by Czestaw BESSAGA

Summary. Suppose that $K$ is a $\mathrm{CW}$-complex, $\mathbf{X}$ is an inverse sequence of stratifiable spaces, and $X=\lim \mathbf{X}$. Using the concept of semi-sequence, we provide a necessary and sufficient condition for $X$ to be an absolute co-extensor for $K$ in terms of the inverse sequence $\mathbf{X}$ and without recourse to any specific properties of its limit. To say that $X$ is an absolute co-extensor for $K$ is the same as saying that $K$ is an absolute extensor for $X$, i.e., that each map $f: A \rightarrow K$ from a closed subset $A$ of $X$ extends to a map $F: X \rightarrow K$. In case $K$ is a polyhedron $|K|_{\mathrm{CW}}$ (the set $|K|$ with the weak topology CW), we determine a similar characterization that takes into account the simplicial structure of $K$.

1. Introduction. The following limit theorem of K. Nagami [13] has been used frequently since its first appearance in 1959 (see [14] for proof details).

THEOREM 1.1. Let $\mathbf{X}=\left(X_{i}, p_{i}^{i+1}\right)$ be an inverse sequence of metrizable spaces, $X=\lim \mathbf{X}$, and suppose that for each $i \in \mathbb{N}, \operatorname{dim} X_{i} \leq n$. Then $\operatorname{dim} X \leq n$.

This theorem, however, can be seen as a result in extension theory (see e.g. [5]) for the following reasons. If $K$ is a CW-complex and $X$ is a space, then one says that $K$ is an absolute extensor for $X, K \in \mathrm{AE}(X)$, or that $X$ is an absolute co-extensor for $K, X \tau K$, if for each closed subset $A$ of $X$ and map (i.e., continuous function) $f: A \rightarrow K$, there exists a map $F: X \rightarrow K$ such that $F$ is an extension of $f$. Since it is well-known that for a metrizable

2000 Mathematics Subject Classification: 54C55, 54B35, 54F45.

Key words and phrases: absolute co-extensor, absolute extensor, absolute neighborhood extensor, canonical map, cohomological dimension, contiguity, CW-complex, Eilenberg-Mac Lane complex, extension theory, inverse limit, inverse sequence, $K$ modification, nerve, polyhedron, semi-sequence, semi-limit, simplicial complex, stratifiable space. 
(or even stratifiable) space $X, \operatorname{dim} X \leq n$ if and only if $X$ is an absolute co-extensor for $S^{n}$, Theorem 1.1 can be stated in the following way.

TheORem 1.2. Let $\mathbf{X}=\left(X_{i}, p_{i}^{i+1}\right)$ be an inverse sequence of metrizable spaces, $X=\lim \mathbf{X}$, and suppose that for each $i \in \mathbb{N}, X_{i}$ is an absolute co-extensor for $S^{n}$. Then $X$ is an absolute co-extensor for $S^{n}$.

A direct generalization of Theorem 1.2 was given by L. Rubin and P. Schapiro in [17]:

TheOREM 1.3. Let $K$ be a $\mathrm{CW}$-complex, $\mathbf{X}=\left(X_{i}, p_{i}^{i+1}\right)$ an inverse sequence of metrizable spaces such that for each $i \in \mathbb{N}, X_{i}$ is an absolute coextensor for $K$, and $X=\lim \mathbf{X}$. Then $X$ is an absolute co-extensor for $K$.

Several generalizations between Theorem 1.2 and Theorem 1.3 are listed in [17]. Theorem 1.3 has already been applied in [4] and [9].

Another step was taken in [16]. Instead of requiring that $X_{i}$ is an absolute co-extensor for $K$ for each $i \in \mathbb{N}$, a condition was placed on the bonding maps $p_{i}^{i+1}$. The requirement was that for each $i \in \mathbb{N}$, closed subset $A$ of $X_{i}$, and map $f: A \rightarrow K$, there are to exist $j \geq i$ and a map $F: X_{j} \rightarrow K$ having the property that $F(x)=f\left(p_{i}^{j}(x)\right)$ for each $x \in\left(p_{i}^{j}\right)^{-1}(A)$. S. Mardešić [10] extended the work in [16] to the class of stratifiable spaces. More recently the notion of semi-sequence was introduced in [7] and a limit theorem in extension theory was proved there for a semi-limit within the inverse limit of an inverse sequence of stratifiable spaces and for arbitrary CW-complexes.

All the previous theorems contain only sufficient conditions for $X \tau K$ when $X=\lim \mathbf{X}$. In this paper, we go further and characterize the existence of the absolute co-extensor property in the limit with respect to a given $\mathrm{CW}$ complex $K$ (Theorem 3.1), or polyhedron $|K|_{\mathrm{CW}}$ (Theorem 3.2), in terms only of the sequence itself.

If $K$ denotes a simplicial complex whose polyhedron is $|K|_{\mathrm{CW}}$, then the characterization Theorem 3.2 takes into account the simplicial structure of $K$. We note here that by $|K|_{\text {CW }}$ we mean the polyhedron with the weak topology $\mathrm{CW}$ induced by the triangulation $K$. Such a polyhedron will also be treated as a CW-complex, its CW-structure coming from the triangulation $K$ in a canonical way. All this is done within the class of stratifiable spaces ([1], [3]) that contains the class of metrizable spaces. This class has many properties convenient for extension theory, like the homotopy extension property with respect to $\mathrm{CW}$ complexes, being hereditarily paracompact, and others $\left({ }^{1}\right)$.

$\left({ }^{1}\right)$ In [8] this characterization is used to prove a limit theorem under a local $\sigma$-type condition on $\mathbf{X}$; one can also find there a list of properties of stratifiable spaces that we use herein. 
Our main results, Theorems 3.1 and 3.2, appear in Section 3, and are stated in terms of semi-sequences and semi-limits. As mentioned above, these concepts were introduced in [7], but we shall shortly provide all that is needed to understand the theorems. We shall define, for example, the notion of a map of a semi-sequence to a space, $K$-modification and contiguity of such maps, and homotopy of such maps.

Theorems 3.1 and 3.2 lead to characterizations (Theorems 3.3 and 3.4) of the absolute co-extensor property for spaces. They are given in terms of pairs of open sets and a map of one of them to the given $\mathrm{CW}$-complex $K$ or polyhedron $|K|_{\mathrm{CW}}$.

Some information about semi-sequences is presented in Section 2, while Section 4 contains our Main Lemma. The proofs of Theorems 3.1 and 3.2 are given in Section 5 .

2. Semi-sequences. In this section we are going to provide a portion of the theory of semi-sequences. For the remainder of this section $\mathbf{X}=$ $\left(X_{i}, p_{i}^{i+1}\right)$ will denote an inverse sequence of spaces and $X=\lim \mathbf{X}$. Let us repeat Definition 1.3 of [7].

Definition 2.1. Let $\mathbb{N}^{*}$ be an infinite subset of $\mathbb{N}$, and for each $i \in \mathbb{N}^{*}$, $M_{i}$ a subset of $X_{i}$. We shall refer to $\mathbf{M}=\left(M_{i}, \mathbb{N}^{*}\right)$ as a semi-sequence of $\mathbf{X}$ and define $\operatorname{sim} \mathbf{M}$ to be those $x \in X$ having the property that there exists $i \in \mathbb{N}^{*}$ such that $x_{j} \in M_{j}$ for all $j \in \mathbb{N}^{*}$ and $j \geq i$. We call $M=\operatorname{slim} \mathbf{M}$ the semi-limit of $\mathbf{M}$.

In this paper, however, we shall always use $\mathbb{N}^{*}=\mathbb{N}$, so let us just write $\mathbf{M}=\left(M_{i}\right)$ instead of $\left(M_{i}, \mathbb{N}\right)$. We may always treat $\mathbf{X}$ as $\left(X_{i}\right)$, i.e., we may think of $\mathbf{X}$ as a semi-sequence of $\mathbf{X}$. As usual, $p_{i}: X=\operatorname{slim} \mathbf{X} \rightarrow X_{i}$ will denote the $i$ th coordinate projection.

Whenever $x \in \operatorname{slim} \mathbf{M}$ then there exists a first $i \in \mathbb{N}$ such that $x_{j} \in M_{j}$ for all $j \geq i$. We shall denote this by $i=\phi_{\mathbf{M}}(x)$ and call it the $\mathbf{M}$-birth index of $x$.

Definition 2.2. Let $\mathbf{M}=\left(M_{i}\right), \mathbf{H}=\left(H_{i}\right)$ be semi-sequences of $\mathbf{X}$.

(1) We shall call $\mathbf{M}$ a subsemi-sequence of $\mathbf{H}$ if for each $i \in \mathbb{N}, M_{i} \subset H_{i}$.

(2) Define the union of $\mathbf{M}$ and $\mathbf{H}, \mathbf{M} \cup \mathbf{H}$, to be the semi-sequence $\left(M_{i} \cup H_{i}\right)$ of $\mathbf{X}$. The intersection $\mathbf{M} \cap \mathbf{H}$ is defined as $\left(M_{i} \cap H_{i}\right)$.

(3) Let us say that $\mathbf{M}$ is open (resp., closed) in $\mathbf{X}$ if for each $i \in \mathbb{N}, M_{i}$ is open (resp., closed) in $X_{i}$.

(4) Call $\mathbf{M}$ an expanding semi-sequence of $\mathbf{X}$ if $\left(p_{i}^{i+1}\right)^{-1}\left(M_{i}\right) \subset M_{i+1}$ for each $i \in \mathbb{N}$. 
(5) Assume that $K$ is a space, and for each $i \in \mathbb{N}, g_{i}: H_{i} \rightarrow K$ is a map. We shall then say that $\mathbf{g}=\left(g_{i}\right)$ is a map of $\mathbf{H}$ to $K$ and denote this by $\mathbf{g}: \mathbf{H} \rightarrow K$ if the consistency equation, $g_{i+1}(x)=g_{i} \circ p_{i}^{i+1}(x)$, is satisfied whenever $x \in\left(p_{i}^{i+1}\right)^{-1}\left(H_{i}\right) \cap H_{i+1}$ and $i \in \mathbb{N}$.

(6) Let $\mathbf{g}=\left(g_{i}\right): \mathbf{H} \rightarrow K, \mathbf{h}=\left(h_{i}\right): \mathbf{M} \rightarrow K$ be maps, $\mathbf{M}$ a subsemisequence of $\mathbf{H}$, and $h_{i}=g_{i} \mid M_{i}: M_{i} \rightarrow K$ for each $i \in \mathbb{N}$. Then we say that $\mathbf{h}$ is the restriction of $\mathbf{g}$ to $\mathbf{M}$, written $\mathbf{h}=\mathbf{g} \mid \mathbf{M}$, and $\mathbf{g}$ is an extension of $\mathbf{h}$ to $\mathbf{H}$.

We shall use $I$ to denote the closed unit interval $[0,1]$. The following example should help the reader gain an understanding of semi-sequences and their semi-limits.

EXAMPLE 2.3. Let $\mathbf{I}=\left(I_{i}, p_{i}^{i+1}\right)$ where for each $i, I_{i}=I$ and $p_{i}^{i+1}$ is the identity map. Put $M_{i}$ equal to the union of the $2^{i-1}$ closed intervals that one uses in the standard construction of the Cantor set and $H_{i}=I_{i} \backslash M_{i}$. Let $\mathbf{M}=\left(M_{i}\right)$ and $\mathbf{H}=\left(H_{i}\right)$. Then of course both $\mathbf{M}$ and $\mathbf{H}$ are semi-sequences of $\mathbf{I}$, and one may even treat the former as an inverse subsequence of $\mathbf{I}$ whose bonding maps are the restrictions of the identity maps, which are just inclusions. Clearly slim $\mathbf{M}$ equals the Cantor set if we identify $\lim \mathbf{I}$ with $I$. But also $\mathbf{H}$ is an expanding open semi-sequence of $\mathbf{I}$, and $\operatorname{sim} \mathbf{H}$ equals the complement of the Cantor set under this identification.

Let us point out the following. Let $\mathbf{M}$ be a semi-sequence of $\mathbf{X}, K$ a space, and $\mathbf{g}=\left(g_{i}\right): \mathbf{M} \rightarrow K$ a map. Then there is a function $g: \operatorname{sim} \mathbf{M} \rightarrow K$ given by $g(x)=g_{i} \circ p_{i}(x)$ for each $x \in \operatorname{sim} \mathbf{M}$ and $i \geq \phi_{\mathbf{M}}(x)$. If in addition, $\alpha: K \rightarrow K_{0}$ is a map, then $\mathbf{h}=\left(\alpha \circ g_{i}\right)$ is a map of $\mathbf{M}$ to $K_{0}$. We shall refer to $g: \operatorname{sim} \mathbf{M} \rightarrow K$ as the semi-limit of $\mathbf{g}$ and denote it by slim $\mathbf{g}$. The map $\mathbf{h}$ will be denoted $\alpha \circ \mathrm{g}$. Many other facts about semi-sequences follow readily from the previous definitions and we shall point them out when needed. Here is a fact of such type.

Lemma 2.4. Let $\mathbf{M}=\left(M_{i}\right)$ be an expanding open semi-sequence of $\mathbf{X}$. For each $i \in \mathbb{N}$, let $U_{i}=p_{i}^{-1}\left(M_{i}\right)$, and put $U=\bigcup\left\{U_{i} \mid i \in \mathbb{N}\right\}$. Then $U=$ $\operatorname{sim} \mathbf{M}$, and $U$ is open in $X$. If $K$ is a space and $\mathbf{g}=\left(g_{i}\right)$ a map of $\mathbf{M}$ to $K$, then $g=\operatorname{slim} \mathrm{g}: U \rightarrow K$ is a map having the property that $g\left|U_{i}=g_{i} \circ p_{i}\right| U_{i}$ for each $i \in \mathbb{N}$.

In [17] we introduced the notion of "response." Let us review it here.

Definition 2.5. Let $f: X \rightarrow Y$ be a map and $W$ be an open subset of $X$. Then $\operatorname{resp}(W, f)$ is the maximal open subset $U$ of $Y$ such that $f^{-1}(U) \subset W$. 
Note that if $W \subset W^{\prime}$ are open subsets of a space $X, f: X \rightarrow Y$, $g: Y \rightarrow Z$ are maps, and $h=g \circ f: X \rightarrow Z$, then

$$
g^{-1}(\operatorname{resp}(W, h)) \subset \operatorname{resp}(W, f) \subset \operatorname{resp}\left(W^{\prime}, f\right) .
$$

Lemma 2.6. Let $\mathbf{X}=\left(X_{i}, p_{i}^{i+1}\right)$ be an inverse sequence of spaces, $X=$ $\lim \mathbf{X}, \mathcal{W}=\left\{W_{v} \mid v \in \Gamma\right\}$ an open collection in $X$, and $W=\bigcup\left\{W_{v} \mid v \in \Gamma\right\}$. For $i \in \mathbb{N}$, set $W_{i, v}=\operatorname{resp}\left(W_{v}, p_{i}\right), v \in \Gamma ; \mathcal{W}_{i}=\left\{W_{i, v} \mid v \in \Gamma\right\}$; and $W_{i}=\bigcup \mathcal{W}_{i}$. Then $\mathbf{W}=\left(W_{i}\right)$ is an expanding open semi-sequence of $\mathbf{X}$ with $W=\operatorname{sim} \mathbf{W}$.

Proof. Clearly $\mathbf{W}$ is open and expanding. Let $x \in W$. Then for some $v \in \Gamma, x \in W_{v}$. There exists $i \in \mathbb{N}$ and a neighborhood $V$ of $x_{i}$ in $X_{i}$ such that $p_{i}{ }^{-1}(V) \subset W_{v}$. Thus $V \subset W_{i, v}$ and we see that $x_{j} \in W_{j, v}$ for all $j \geq i$. Therefore $W \subset \operatorname{slim} \mathbf{W}$. On the other hand, suppose that $x \in \operatorname{slim} \mathbf{W}$. Let $i=\Phi_{\mathbf{W}}(x)$, the birth index of $x$. Then $x_{i} \in W_{i}$, so $x \in p_{i}^{-1}\left(W_{i}\right)=$ $\bigcup\left\{p_{i}^{-1}\left(W_{i, v}\right) \mid v \in \Gamma\right\} \subset W$, due to the definition of response.

In such cases we shall refer to $\mathbf{W}$ as the expanding open semi-sequence of $\mathbf{X}$ induced by $\mathcal{W}$.

Let $\mathbf{Y}=\left(Y_{i}, q_{i}^{i+1}\right)$ be an inverse sequence of spaces such that for each $i \in \mathbb{N}, X_{i} \subset Y_{i}$ and $p_{i}^{i+1}=q_{i}^{i+1} \mid X_{i}$. Then we shall say that $\mathbf{X}$ is an inverse subsequence of $\mathbf{Y}$. In case $\mathbf{M}$ is a semi-sequence of $\mathbf{X}$, we may also treat it as a semi-sequence of $\mathbf{Y}$. In this case, we shall distinguish the semi-limits by writing $\operatorname{slim}_{\mathbf{X}} \mathbf{M}$ and $\operatorname{slim}_{\mathbf{Y}} \mathbf{M}$, even though one might treat them as being equal.

LEMma 2.7. Let $\mathbf{X}=\left(X_{i}, p_{i}^{i+1}\right)$ be an inverse subsequence of an inverse sequence $\mathbf{Y}=\left(Y_{i}, q_{i}^{i+1}\right), X=\lim \mathbf{X}$, and $Y=\lim \mathbf{Y}$. Suppose that $\mathbf{E}$ is a semi-sequence of $\mathbf{Y}$. Then:

(1) $\operatorname{sim}_{\mathbf{Y}}(\mathbf{E} \cap \mathbf{X}) \subset X$ and $\operatorname{sim}_{\mathbf{Y}}(\mathbf{E} \cap \mathbf{X})=\operatorname{sim}_{\mathbf{X}}(\mathbf{E} \cap \mathbf{X})$,

(2) $\left(\operatorname{sim}_{\mathbf{Y}} \mathbf{E}\right) \cap X=\operatorname{sim}_{\mathbf{X}}(\mathbf{E} \cap \mathbf{X})$,

(3) if $\mathbf{E}$ is expanding in $\mathbf{Y}$, then $\mathbf{E} \cap \mathbf{X}$ is expanding in $\mathbf{X}$,

(4) if $\mathbf{E}$ is open (resp. closed) in $\mathbf{Y}$, then $\mathbf{E} \cap \mathbf{X}$ is open (resp. closed) in $\mathbf{X}$.

In the proof of Proposition 1.7 of [16] the author performed a construction from which the following fact follows.

LeMma 2.8. Let $\mathbf{X}=\left(X_{i}, p_{i}^{i+1}\right)$ be an inverse sequence of spaces and $X=\lim \mathbf{X}$. Then there exists an inverse sequence $\widetilde{\mathbf{X}}=\left(\widetilde{X}_{i}, \widetilde{p}_{i}^{i+1}\right)$ of spaces and surjective bonding maps with $\widetilde{X}=\lim \widetilde{\mathbf{X}}$ such that for each $i \in \mathbb{N}, \widetilde{X}_{i}=$ $X_{i}+D_{i}$ where $D_{i}$ is a discrete subspace of $\widetilde{X}_{i}, D_{1}=\emptyset, \widetilde{p}_{i}^{i+1} \mid X_{i+1}=p_{i}^{i+1}$, and for each $x \in D_{i+1},\left(\widetilde{p}_{i}^{i+1}\right)^{-1}\left\{\widetilde{p}_{i}^{i+1}(x)\right\}=\{x\}$. In this case:

(1) $\mathbf{X}=\left(X_{i}\right)$ is an open semi-sequence of $\widetilde{\mathbf{X}}$, 
(2) $\mathbf{D}=\left(D_{i}\right)$ is an expanding open semi-sequence of $\widetilde{\mathbf{X}}$,

(3) $\operatorname{sim} \mathbf{D}=\tilde{X} \backslash X$,

(4) for each $i \in \mathbb{N}$ and $x \in \widetilde{p}_{i}^{i+1}\left(D_{i+1}\right)$, there is a unique $x^{i+1} \in D_{i+1}$ with $\tilde{p}_{i}^{i+1}\left(x^{i+1}\right)=x$,

(5) $\tilde{X} \backslash X$ is a discrete open subspace of $\tilde{X}$,

(6) $X=\operatorname{slim}_{\mathbf{X}} \mathbf{X}$ and $X$ is a closed subspace of $\tilde{X}$.

Let us refer to $\widetilde{\mathbf{X}}$ as a surjective extension of $\mathbf{X}$. If $X_{i}$ is stratifiable for each $i \in \mathbb{N}$, then $\widetilde{X}$ is stratifiable. Furthermore, $\widetilde{X} \tau K$ if and only if $X \tau K$.

We shall denote by $\mathbf{X} \times I$ the inverse sequence $\left(X_{i} \times I, p_{i}^{i+1} \times \mathrm{id}\right)$. We of course identify $\lim (\mathbf{X} \times I)$ with $X \times I$. If $\mathbf{M}=\left(M_{i}\right)$ is a semi-sequence of $\mathbf{X}$, then we shall use $\mathbf{M} \times I$ to denote the semi-sequence $\left(M_{i} \times I\right)$ of $\mathbf{X} \times I$. If we put $M=\operatorname{sim} \mathbf{M}$, then again $\operatorname{sim}(\mathbf{M} \times I)$ may be thought of as $M \times I \subset X \times I$.

Definition 2.9. Let $\mathbf{F}=\left(F_{i}\right): \mathbf{M} \times I \rightarrow Y$ be a map. For each $i \in \mathbb{N}$, let $g_{i}: M_{i} \rightarrow Y$ be given by $g_{i}(x)=F_{i}(x, 0)$ and $h_{i}: M_{i} \rightarrow Y$ be given by $h_{i}(x)=F_{i}(x, 1)$. Then $\mathbf{g}=\left(g_{i}\right)$ and $\mathbf{h}=\left(h_{i}\right)$ are maps of $\mathbf{M}$ to $Y$. We shall say that $\mathbf{F}$ is a homotopy from $\mathbf{g}$ to $\mathbf{h}$. Under such conditions, we will write $\mathbf{g} \simeq \mathbf{h}$ and say that $\mathbf{g}$ is homotopic to $\mathbf{h}$.

It is clear that $\simeq$ is an equivalence relation on the set of maps $\mathrm{g}$ : $\mathbf{M} \rightarrow Y$. Furthermore, under some additional conditions homotopic maps induce homotopic maps of the semi-limit.

LEMMA 2.10. If $\mathbf{M}$ is expanding open and $\mathbf{F}$ is a homotopy from $\mathbf{g}$ to $\mathbf{h}$, then $F=\operatorname{sim} \mathbf{F}: M \times I \rightarrow Y, g=\operatorname{slim} \mathbf{g}: M \rightarrow Y$, and $h=\operatorname{slim} \mathbf{h}: M \rightarrow Y$ are maps, and $F$ is a homotopy from $g$ to $h$.

Let us recall the notions of $K$-modification and contiguity. Let $K$ be a simplicial complex and $g, h: C \rightarrow|K|_{\mathrm{CW}}$ be maps. Then we say that $g$ is a $K$-modification of $h$ if for each $x \in C$, whenever $\sigma \in K$ and $h(x) \in \sigma$, then $g(x) \in \sigma$. On the other hand, one says that $g$ is contiguous to $h$ if for each $x \in C$, there exists $\sigma \in K$ such that $g(x), h(x) \in \sigma\left({ }^{2}\right)$.

Note that $g$ is a $K$-modification of $h$ if and only if for each $x \in C$ and $\sigma \in K$ such that $h(x) \in \operatorname{int} \sigma$, we have $g(x) \in \sigma$. Recall that if $g$ is a $K$-modification of $h$, then $g$ is contiguous to $h$, and that contiguous maps are homotopic.

Next are our definitions of $K$-modification and contiguity for maps of semi-sequences.

$\left({ }^{2}\right)$ When $g$ is contiguous to $h$, it need not be true that either one of them is a $K$-modification of the other. Also contiguity is not an equivalence relation although it is reflexive and symmetric. 
Definition 2.11. Let $\mathbf{g}=\left(g_{i}\right)$ and $\mathbf{h}=\left(h_{i}\right)$ be maps of $\mathbf{M}$ to a polyhedron $|K|_{\mathcal{T}}$. Define $\mathbf{g}$ to be a $K$-modification of $\mathbf{h}$ if $g_{i}$ is a $K$-modification of $h_{i}$ for each $i \in \mathbb{N}$. We shall say that $\mathbf{g}$ is contiguous to $\mathbf{h}$ if $g_{i}$ is contiguous to $h_{i}$ for each $i \in \mathbb{N}$.

From our definitions it easily follows that if $\mathbf{g}$ is a $K$-modification of $\mathbf{h}$, then $\mathbf{g}$ is contiguous to $\mathbf{h}$.

Let us now quote Lemma 2.3 of [12], noting that this is a generalization of Theorem 10, page 302 of [11].

Lemma 2.12. Let $K$ be a simplicial complex and $\mathcal{T}$ a paracompact topology for $|K|$ such that:

(1) $\mathcal{T} \subset \mathrm{CW}$,

(2) for each $\sigma \in K, \sigma_{\mathcal{T}}=\sigma_{\mathrm{CW}}$,

(3) for each $v \in K^{(0)}, \operatorname{st}(v, K)$ is open in $|K|_{\mathcal{T}}$.

Let $i_{1}:|K| \rightarrow|K|$ be the identity function and $i_{2}:|K|_{\mathrm{CW}} \rightarrow|K|_{\mathcal{T}}$ be the identity map. Then there is a map $j:|K|_{\mathcal{T}} \rightarrow|K|_{\mathrm{CW}}$ which is a $K$ modification of $i_{1}$ and such that $j$ is a homotopy equivalence with homotopy inverse $i_{2}$. In fact, there is a function $H:|K| \times I \rightarrow|K|$ such that for each $t \in I, H_{t}:|K| \rightarrow|K|$ is simplex preserving and:

(a) $H:|K|_{\mathrm{CW}} \times I \rightarrow|K|_{\mathrm{CW}}$ is a homotopy between $j \circ i_{2}$ and the identity on $|K|_{\mathrm{CW}}$,

(b) $H:|K|_{\mathcal{T}} \times I \rightarrow|K|_{\mathcal{T}}$ is a homotopy between $i_{2} \circ j$ and the identity on $|K|_{\mathcal{T}}$.

Lemma 2.13. Let $\mathbf{g}=\left(g_{i}\right)$ and $\mathbf{h}=\left(h_{i}\right)$ be maps of $\mathbf{M}$ to a polyhedron $|K|_{\mathrm{CW}}$. If $\mathbf{g}$ is contiguous to (or is a K-modification of) $\mathbf{h}$, then $\mathbf{g} \simeq \mathbf{h}$.

Proof. Let $\mathcal{T}$ designate the metric topology on $|K|$ induced by the triangulation $K$ (as in Appendix I of [11]). Since open vertex stars belong to $\mathcal{T}$, one sees that $\mathcal{T}$ meets the requirements of Lemma 2.12 , so let $i_{1}, i_{2}, j$, and $H$ be as in that lemma.

Fix $i \in \mathbb{N}$. The map $i_{2} \circ g_{i}$ is contiguous to $i_{2} \circ h_{i}$ from $M_{i}$ to $|K|_{\mathcal{T}}$. The so-called "straight line" homotopy $G_{i}$ of these maps is given as follows. If $x \in M_{i}, v \in K^{(0)}, a$ is the $v$-barycentric coordinate of $i_{2} \circ g_{i}(x), b$ is the $v$-barycentric coordinate of $i_{2} \circ h_{i}(x)$, and $t \in I$, then $(1-t) a+t b$ is the $v$-barycentric coordinate of $G_{i}(x, t)$. This function $G_{i}: M_{i} \times I \rightarrow|K|_{\mathcal{T}}$ is continuous since all its barycentric coordinates are continuous (Appendix I of [11]). One then sees that $j \circ G_{i}: M_{i} \times I \rightarrow|K|_{\mathrm{CW}}$ is a homotopy from $j \circ i_{2} \circ g_{i}$ to $j \circ i_{2} \circ h_{i}$.

Now consider the homotopy $H$ from (a) in Lemma 2.12, and let $F$ be its reverse, i.e., $F(x, t)=H(x, 1-t)$. Using $F$ we get a homotopy from $\operatorname{id}_{\mathrm{CW}} \circ g_{i}=g_{i}$ to $j \circ i_{2} \circ g_{i}$. Next apply the homotopy $G_{i}$ to go from $j \circ i_{2} \circ g_{i}$ 
to $j \circ i_{2} \circ h_{i}$. Finally, apply again the homotopy $H$ in (a) to go from $j \circ i_{2} \circ h_{i}$ to $\operatorname{id}_{\mathrm{CW}} \circ h_{i}=h_{i}$.

Since the homotopy $G_{i}$ is based on the "straight line" homotopy and $F, H$ are fixed throughout, it is not difficult to see that the consistency relations hold. This completes our proof.

3. Characterization theorems. We now present our characterization theorems, the main results of this paper. There is a $\mathrm{CW}$-version when the target is a CW-complex and an SC-version when the target is a polyhedron.

Theorem 3.1 (CW-version). Let $\mathbf{X}=\left(X_{i}, p_{i}^{i+1}\right)$ be an inverse sequence of stratifiable spaces, $X=\lim \mathbf{X}$, and $K$ be a $C W$-complex. Then $X$ is an absolute co-extensor for $K$ if and only if for any expanding open semisequences $\mathbf{M}$ and $\mathbf{H}$ of $\mathbf{X}$ and map $\mathbf{g}: \mathbf{M} \rightarrow K$, there exist expanding open subsemi-sequences, $\mathbf{M}^{*}$ of $\mathbf{M}$ and $\mathbf{H}^{*}$ of $\mathbf{H}$, and a map $\mathbf{g}^{*}: \mathbf{M}^{*} \cup \mathbf{H}^{*} \rightarrow K$ such that:

(1) $\operatorname{sim} \mathbf{M}^{*} \cup \operatorname{slim} \mathbf{H}^{*}=\operatorname{sim} \mathbf{M} \cup \operatorname{sim} \mathbf{H}$,

(2) $\mathbf{g}^{*}\left|\mathbf{M}^{*} \simeq \mathbf{g}\right| \mathbf{M}^{*}$.

TheOREM 3.2 (SC-version). Let $\mathbf{X}=\left(X_{i}, p_{i}^{i+1}\right)$ be an inverse sequence of stratifiable spaces, $X=\lim \mathbf{X}$, and $K$ be a simplicial complex. Then $X$ is an absolute co-extensor for $|K|_{\mathrm{CW}}$ if and only if for any expanding open semisequences $\mathbf{M}$ and $\mathbf{H}$ of $\mathbf{X}$ and map $\mathbf{g}: \mathbf{M} \rightarrow|K|_{\mathrm{CW}}$, there exist expanding open subsemi-sequences, $\mathbf{M}^{*}$ of $\mathbf{M}$ and $\mathbf{H}^{*}$ of $\mathbf{H}$, and a map $\mathbf{g}^{*}: \mathbf{M}^{*} \cup \mathbf{H}^{*} \rightarrow$ $|K|_{\text {CW }}$ such that:

(1) $\operatorname{sim} \mathbf{M}^{*} \cup \operatorname{sim} \mathbf{H}^{*}=\operatorname{sim} \mathbf{M} \cup \operatorname{sim} \mathbf{H}$,

(2) $\mathbf{g}^{*} \mid \mathbf{M}^{*}$ is a $K$-modification of $\mathbf{g} \mid \mathbf{M}^{*}$.

These theorems lead to characterizations of the absolute co-extensor property on the level of stratifiable spaces in terms of pairs of open sets and a map on one of them. We state both versions and prove only the $\mathrm{CW}$-version.

Theorem 3.3. Let $X$ be a stratifiable space and $K$ a CW-complex. Then $X$ is an absolute co-extensor for $K$ if and only if for each pair $M, H$ of open subsets of $X$ and map $g: M \rightarrow K$, there exist open subsets $M^{*}, H^{*}$ of $X$, $M^{*} \subset M, H^{*} \subset H$, and a map $g^{*}: M^{*} \cup H^{*} \rightarrow K$ such that:

(1) $M^{*} \cup H^{*}=M \cup H$,

(2) $g^{*} \mid M^{*}$ is homotopic to $g \mid M^{*}$.

Proof. $(\Rightarrow)$. Note that $M \cup H$ is a normal space. By the shrinking theorem, choose an open subset $M^{*}$ of $M \cup H$ such that $M^{*} \subset \operatorname{cl}_{M \cup H} M^{*} \subset M$ and $M^{*} \cup H=M \cup H$. Put $H^{*}=H$. Then (1) is true. Also $M \cup H$ is an absolute co-extensor for $K$ [6]; hence $g \mid \operatorname{cl}_{M \cup H} M^{*}: \operatorname{cl}_{M \cup H} M^{*} \rightarrow K$ extends 
to a map $g^{*}: M \cup H \rightarrow K$. Since $M^{*} \subset \operatorname{cl}_{M \cup H} M^{*}$, we have $g^{*}\left|M^{*}=g\right| M^{*}$, showing that $(2)$ is true.

$(\Leftarrow)$. Let $A$ be a closed subset of $X$ and $g: A \rightarrow K$ a map. Since $K \in \operatorname{ANE}(X)([2])$, there exists a neighborhood $M$ of $A$ and a map of $M$ to $K$ extending $g$. We shall use $g: M \rightarrow K$ to denote such a map. Put $H=X \backslash A$. Then $H$ is open and $X=M \cup H$.

For each $i \in \mathbb{N}$, let $X_{i}=X, p_{i}^{i+1}=\mathrm{id}, M_{i}=M, H_{i}=H$, and $g_{i}=g$ : $M_{i} \rightarrow K$. Thus $\mathbf{X}=\left(X_{i}, p_{i}^{i+1}\right)$ is an inverse sequence of stratifiable spaces whose limit is $X$. Put $\mathbf{M}=\left(M_{i}\right), \mathbf{H}=\left(H_{i}\right)$, and $\mathbf{g}=\left(g_{i}\right)$. Then $\mathbf{M}, \mathbf{H}$ are expanding open semi-sequences of $\mathbf{X}$ and $\mathbf{g}: \mathbf{M} \rightarrow K$ is a map. We may apply Theorem 3.1 to $(\mathbf{M}, \mathbf{H}, \mathbf{g})$ to obtain $\mathbf{M}^{*}, \mathbf{H}^{*}$, and $\mathbf{g}^{*}$ meeting the conditions stated there. Let $M^{*}=\operatorname{sim} \mathbf{M}^{*}$ and $H^{*}=\operatorname{sim} \mathbf{H}^{*}$. Then $M^{*} \subset M, H^{*} \subset H$, and Lemma 2.4 shows that $M^{*}, H^{*}$ are open in $X$, and that $\operatorname{sim} \mathbf{g}^{*}=g^{*}: M^{*} \cup H^{*}=M \cup H \rightarrow K$ is a map. From $H \cap A=\emptyset$ and $M^{*} \cup H=X$, it follows that $A \subset M^{*}$. Since $\mathbf{g}^{*}\left|\mathbf{M}^{*} \simeq \mathbf{g}\right| \mathbf{M}^{*}$, Lemma 2.10 gives $g^{*}\left|M^{*} \simeq g\right| M^{*}$.

THEOREM 3.4. Let $X$ be a stratifiable space and $K$ a simplicial complex. Then $X$ is an absolute co-extensor for $|K|_{\mathrm{CW}}$ if and only if for each pair $M, H$ of open subsets of $X$ and map $g: M \rightarrow K$, there exist open subsets $M^{*}, H^{*}$ of $X, M^{*} \subset M, H^{*} \subset H$, and a map $g^{*}: M^{*} \cup H^{*} \rightarrow|K|_{\mathrm{CW}}$ such that:

(1) $M^{*} \cup H^{*}=M \cup H$,

(2) $g^{*} \mid M^{*}$ is a $K$-modification of $g \mid M^{*}$.

4. Lemmas. In order to prove Theorem 3.2 we need some lemmas. Let $\mathcal{U}=\left\{U_{v} \mid v \in \Gamma\right\}$ be an open cover of a space $X, N(\mathcal{U})$ its nerve, and $f: X \rightarrow|N(\mathcal{U})|_{\mathrm{CW}}$ be a map. Then we say that $f$ is a locally finite $\mathcal{U}$ canonical map if $f$ is $\mathcal{U}$-canonical and $\left\{f^{-1}\left(\operatorname{st}\left(U_{v}, N(\mathcal{U})\right)\right) \mid v \in \Gamma\right\}$ is a locally finite open cover of $X$. Notice that if $X$ is a paracompact space, then there is a locally finite $\mathcal{U}$-canonical map $f: X \rightarrow|N(\mathcal{U})|_{\mathrm{CW}}$. In the proof of the Main Lemma (Lemma 4.3) we shall need an enhanced version of Lemma 3.8 of [7]:

Lemma 4.1. Let $X$ be a space, $Z$ a closed subset of $X$, and $Z \subset U \subset X$. Suppose we are given a stratifiable space $C$, a map $p: C \rightarrow X, Z_{0} \subset$ $\left\{t \in Z \mid p^{-1}(t) \neq \emptyset\right\}, \Gamma^{*} \subset \Gamma$, and the following data:

(1) a cover $\mathcal{U}=\left\{U_{v} \mid v \in \Gamma^{*}\right\}$ of $U$ by sets open in $U$,

(2) an open cover $\mathcal{V}=\left\{V_{v} \mid v \in \Gamma\right\}$ of $C$ such that $p^{-1}\left(U_{v}\right) \subset V_{v}$ for each $v \in \Gamma^{*}$,

(3) a locally finite $\mathcal{U}$-canonical map $g: U \rightarrow|N(\mathcal{U})|_{\mathrm{CW}}$.

Let $N_{0}$ be the minimal subcomplex of $N(\mathcal{U})$ such that $g\left(Z_{0}\right) \subset\left|N_{0}\right|$. Then there exist a locally finite $\mathcal{U}$-canonical map $h: U \rightarrow|N(\mathcal{U})|_{\mathrm{CW}}$ such that 
$h^{-1}\left(\left|N_{0}\right|\right)$ is a closed neighborhood of $g^{-1}\left(\left|N_{0}\right|\right)$ in $U$ and $h \mid g^{-1}\left(\left|N_{0}\right|\right)=$ $g \mid g^{-1}\left(\left|N_{0}\right|\right)$, a locally finite $\mathcal{V}$-canonical map $g_{0}: C \rightarrow|N(\mathcal{V})|$, and a simplicial injection $\alpha: N_{0} \rightarrow N(\mathcal{V}), \alpha\left(U_{v}\right)=V_{v}$ for all $U_{v} \in N_{0}^{(0)}$, so that $\alpha \circ h \circ p(z)=g_{0}(z)$ for each $z \in P=p^{-1}\left(h^{-1}\left(\left|N_{0}\right|\right)\right)$.

Suppose in addition that $Z^{*} \subset C, p\left(Z^{*}\right)=Z_{0}, \Gamma_{0} \subset \Gamma^{*}$ and $\left\{U_{v} \mid v \in \Gamma_{0}\right\}$ is the set of vertices of a simplex of $N_{0}$. Then $\bigcap\left\{V_{v} \mid v \in \Gamma_{0}\right\} \cap Z^{*} \neq \emptyset$.

Proof. There is no loss of generality in assuming that $\mathcal{U}$ is a locally finite cover of $U$. Using a closed, regular neighborhood $R$ of $\left|N_{0}\right|$ in $|N(\mathcal{U})|_{\mathrm{CW}}$, choose a map $r:|N(\mathcal{U})|_{\mathrm{CW}} \rightarrow|N(\mathcal{U})|_{\mathrm{CW}}$ such that $r$ retracts $R$ to $\left|N_{0}\right|$, and whenever $x$ lies in a simplex $\sigma$ of $N(\mathcal{U})$, then $r(x) \in \sigma$, i.e., $r$ is an $N(\mathcal{U})$ modification of the identity on $|N(\mathcal{U})|_{\mathrm{CW}}$. We ask the reader to check that $h=r \circ g: U \rightarrow|N(\mathcal{U})|_{\mathrm{CW}}$ is a locally finite $\mathcal{U}$-canonical map. Clearly $g^{-1}(R)$ is a closed neighborhood of $g^{-1}\left(\left|N_{0}\right|\right)$ in $U$, and $g^{-1}(R) \subset h^{-1}\left(\left|N_{0}\right|\right)=$ $g^{-1}\left(r^{-1}\left(\left|N_{0}\right|\right)\right)$. Also, $h(t)=g(t)$ for all $t \in g^{-1}\left(\left|N_{0}\right|\right)$.

Let $\mathcal{E}=\left\{E_{v}=V_{v} \cap P \mid v \in \Gamma\right\}$ and $\theta: N(\mathcal{E}) \rightarrow N(\mathcal{V})$ be the simplicial injection determined by the vertex map $E_{v} \mapsto V_{v}$. Suppose that $\Gamma_{0} \subset \Gamma^{*}$ is finite and $\left\{U_{v} \mid v \in \Gamma_{0}\right\}$ is the vertex set of a simplex of $N_{0}$. Applying Lemma 3.7 of [7], let $t \in \bigcap\left\{U_{v} \mid v \in \Gamma_{0}\right\} \cap Z_{0} \subset Z_{0} \subset h^{-1}\left(\left|N_{0}\right|\right)$. Now $p^{-1}(t) \neq \emptyset$, $p^{-1}(t) \subset P$, and $p^{-1}\left(U_{v}\right) \subset V_{v}$ for each $v \in \Gamma_{0}$. Hence $\emptyset \neq p^{-1}(t) \subset \bigcap\left\{E_{v}=\right.$ $\left.V_{v} \cap P \mid v \in \Gamma_{0}\right\}$, showing that $\left\{E_{v} \mid v \in \Gamma_{0}\right\}$ is the vertex set of a simplex of $N(\mathcal{E})$. Therefore the vertex map $\beta\left(U_{v}\right)=E_{v}$ induces a simplicial injection $\beta: N_{0} \rightarrow N(\mathcal{E})$.

Let $f: P \rightarrow|N(\mathcal{E})|$ be given by the rule $f(x)=\beta \circ h \circ p(x)$. We want to show that $f$ is a locally finite $\mathcal{E}$-canonical map. If a vertex $E_{v}$ of $N(\mathcal{E})$ does not lie in the image of $\beta$, then $\beta^{-1}\left(\operatorname{st}\left(E_{v}, N(\mathcal{E})\right)\right)=\emptyset$, so we need only concern ourselves with a vertex $E_{v}$ of $N(\mathcal{E})$ such that $\beta\left(U_{v}\right)=E_{v}$. Surely $\beta^{-1}\left(\operatorname{st}\left(E_{v}, N(\mathcal{E})\right)\right) \subset \operatorname{st}\left(U_{v}, N(\mathcal{U})\right)$. Since $h$ is $\mathcal{U}$-canonical, we have $h^{-1}\left(\operatorname{st}\left(U_{v}, N(\mathcal{U})\right)\right) \subset U_{v}$. Now $(2)$ shows that $p^{-1}\left(U_{v}\right) \subset V_{v}$, so $p^{-1}\left(U_{v}\right) \cap P \subset$ $V_{v} \cap P=E_{v}$ as needed to show that $f$ is $\mathcal{E}$-canonical.

For the locally finite part, let $y \in P$ and $x=p(y)$. Because of (3), there exists a finite subset $\Gamma_{0}$ of $\Gamma^{*}$ and a neighborhood $Q$ of $x$ in $X$ such that $Q \cap h^{-1}\left(\operatorname{st}\left(U_{v}, N(\mathcal{U})\right)\right) \neq \emptyset$ only if $v \in \Gamma_{0}$. So $p^{-1}(Q) \cap P$ is a neighborhood of $y$ in $P$ that intersects $(h \circ p)^{-1}\left(\operatorname{st}\left(U_{v}, N(\mathcal{U})\right)\right) \cap P$ only if $v \in \Gamma_{0}$. From the argument preceding this, one sees that $(\beta \circ h \circ p)^{-1}\left(\operatorname{st}\left(E_{v}, N(\mathcal{E})\right)\right) \subset$ $(h \circ p)^{-1}\left(\operatorname{st}\left(U_{v}, N(\mathcal{U})\right)\right)$ for each $v \in \Gamma$. So the neighborhood $p^{-1}(Q) \cap P$ of $y$ in $P$ intersects $f^{-1}\left(\operatorname{st}\left(E_{v}, N(\mathcal{E})\right)\right)$ only if $v \in \Gamma_{0}$. This completes the last step in showing that $f$ is a locally finite $\mathcal{E}$-canonical map.

Observe that $P$ is a closed subspace of the stratifiable space $C$. Apply Lemma 3.6 of [7] to get a locally finite $\mathcal{V}$-canonical map $g_{0}: C \rightarrow|N(\mathcal{V})|$ so that for $z \in P, \theta(f(z))=g_{0}(z)$. Now $\theta \circ f(z)=\theta \circ \beta \circ h \circ p(z)$, and $\alpha=\theta \circ \beta$ is a simplicial injection. 
To obtain the final statement of our lemma, one should recall that we earlier showed that there exists $t \in \bigcap\left\{U_{v} \mid v \in \Gamma_{0}\right\} \cap Z_{0}$. Choose $c \in Z^{*}$ with $p(c)=t$. Then $c \in p^{-1}\left(U_{v}\right) \subset V_{v}$ for all $v \in \Gamma_{0}$. This completes our proof.

Before getting to our Main Lemma, let us restate in terms of semisequences a fact which appears as Lemma 3 of [10]. We recall here that stratifiable spaces are perfectly normal, i.e., every open subset is an $\mathrm{F}_{\sigma}$-set.

LEMMA 4.2. Let $\mathbf{X}=\left(X_{i}, p_{i}^{i+1}\right)$ be an inverse sequence of stratifiable spaces and $\mathbf{W}=\left(W_{i}\right)$ an expanding open semi-sequence of $\mathbf{X}$. Then there exists an expanding open subsemi-sequence $\mathbf{S}=\left(S_{i}\right)$ of $\mathbf{W}$ such that:

(1) $\operatorname{cl}_{X_{i}}\left(S_{i}\right) \subset W_{i}$ and $\left(p_{i}^{i+1}\right)^{-1}\left(\operatorname{cl}_{X_{i}}\left(S_{i}\right)\right) \subset S_{i+1}$ for each $i \in \mathbb{N}$,

(2) $\operatorname{sim} \mathbf{S}=\operatorname{sim} \mathbf{W}$.

Next we present our Main Lemma. It is worth mentioning that the proof of this lemma requires a target space that is a polyhedron. We shall need Lemma 4.3 in the proof of Theorem 3.2.

Lemma 4.3. Let $\mathbf{X}=\left(X_{i}, p_{i}^{i+1}\right)$ be an inverse sequence of stratifiable spaces, $K$ a simplicial complex, $W$ an open subset of $X=\lim \mathbf{X}$, and $f$ : $W \rightarrow|K|_{\mathrm{CW}}$ a map. Then there exist:

(1) an expanding open semi-sequence $\mathbf{M}=\left(M_{i}\right)$ of $\mathbf{X}$ with $\operatorname{sim} \mathbf{M}=W$,

(2) a map $\mathbf{g}$ of $\mathbf{M}$ to $|K|_{\mathrm{CW}}$ such that the map $g=\operatorname{sim} \mathbf{g}: W \rightarrow|K|_{\mathrm{CW}}$ is a $K$-modification of $f$.

Proof. Let $\Gamma=K^{(0)}$, for each $v \in \Gamma, W_{v}=f^{-1}(\operatorname{st}(v, K)), \mathcal{W}=$ $\left\{W_{v} \mid v \in \Gamma\right\}$, and $\mathbf{W}=\left(W_{i}\right)$ be the expanding open semi-sequence of $\mathbf{X}$ induced by $\mathcal{W}$ (see after Lemma 2.6). Then $W=\operatorname{sim} \mathbf{W}$.

(F1) The vertex map $W_{v} \mapsto v, v \in \Gamma$, determines a simplicial injection $\kappa: N(\mathcal{W}) \rightarrow K$.

Apply Lemma 4.2 to obtain $\mathbf{S}=\left(S_{i}\right)$, an expanding open subsemi-sequence of $\mathbf{W}$ such that $\operatorname{sim} \mathbf{S}=\operatorname{sim} \mathbf{W}=W$, and for each $i \in \mathbb{N}$, both $\operatorname{cl}_{X_{i}}\left(S_{i}\right) \subset$ $W_{i}$ and $\left(p_{i}^{i+1}\right)^{-1}\left(\operatorname{cl}_{X_{i}}\left(S_{i}\right)\right) \subset S_{i+1}$. For each $i \in \mathbb{N}$, put:

$$
Z_{i}=p_{i}(W) \cap \operatorname{cl}_{X_{i}}\left(S_{i}\right) .
$$

We want to establish the following fact:

(F3) Let $i \in \mathbb{N}, s: W_{i} \rightarrow\left|N\left(\mathcal{W}_{i}\right)\right|$ be a $\mathcal{W}_{i}$-canonical map, and $N_{0}$ the minimal subcomplex of $N\left(\mathcal{W}_{i}\right)$ such that $s\left(Z_{i}\right) \subset\left|N_{0}\right|$. Suppose that $\Gamma_{0} \subset \Gamma$ is finite and $\left\{W_{i, v} \mid v \in \Gamma_{0}\right\}$ is the set of vertices of a simplex of $N_{0}$. Then $\left\{W_{v} \mid v \in \Gamma_{0}\right\}$ is the set of vertices of a simplex of $N(\mathcal{W})$.

Indeed, by Lemma 3.7 of [7], there exists $t \in \bigcap\left\{U_{i, v} \mid v \in \Gamma_{0}\right\} \cap Z_{i}$. So there is $w \in W$ with $p_{i}(w)=t$. Since $W_{i, v}=\operatorname{resp}\left(p_{i}, W_{v}\right)$, it follows that $w \in p_{i}^{-1}\left(W_{i, v}\right) \subset W_{v}$ for all $v \in \Gamma_{0}$.

Let us note that 
(F4) for each $i<j$ in $\mathbb{N}, Z_{i} \subset p_{i}^{j}\left(Z_{j}\right)$.

To see this, let $z \in Z_{i}$. Then for some $w \in W, z=p_{i}(w) \in \operatorname{cl}_{X_{i}}\left(S_{i}\right)$. Now $z=p_{i}^{j} \circ p_{j}(w)$, so $p_{j}(w) \in\left(p_{i}^{j}\right)^{-1}(z) \subset\left(p_{i}^{j}\right)^{-1}\left(\mathrm{cl}_{X_{i}}\left(S_{i}\right)\right) \subset S_{j}$. Hence $p_{j}(w) \in Z_{j}=p_{j}(W) \cap \mathrm{cl}_{X_{j}}\left(S_{j}\right)$.

Select a locally finite $\mathcal{W}_{1}$-canonical map $s_{1}: W_{1} \rightarrow\left|N\left(\mathcal{W}_{1}\right)\right|_{\text {CW. Define }}$ $N_{1}$ to be the minimum subcomplex of $N\left(\mathcal{W}_{1}\right)$ such that $s_{1}\left(Z_{1}\right) \subset\left|N_{1}\right|$. Suppose that $i \in \mathbb{N}$ and for each $1 \leq k \leq i$ we have determined:

(I1) a locally finite $\mathcal{W}_{k}$-canonical map $s_{k}: W_{k} \rightarrow\left|N\left(\mathcal{W}_{k}\right)\right|_{\mathrm{CW}}$ and have designated by $N_{k}$ the minimal subcomplex of $N\left(\mathcal{W}_{k}\right)$ with $s_{k}\left(Z_{k}\right) \subset\left|N_{k}\right|$,

(I2) if $k<i$, a locally finite $\mathcal{W}_{k}$-canonical map $h_{k}: W_{k} \rightarrow\left|N\left(\mathcal{W}_{k}\right)\right|_{\text {CW }}$ such that $h_{k}^{-1}\left(\left|N_{k}\right|\right)$ is a closed neighborhood of $s_{k}^{-1}\left(\left|N_{k}\right|\right)$ and $h_{k}\left|s_{k}^{-1}\left(\left|N_{k}\right|\right)=s_{k}\right| s_{k}^{-1}\left(\left|N_{k}\right|\right)$,

(I3) if $k<i$, a simplicial injection $\alpha_{k}: N_{k} \rightarrow N_{k+1}, \alpha_{k}\left(W_{k, v}\right)=W_{k+1, v}$ for each $W_{k, v} \in\left(N_{k}\right)^{(0)}$, such that $\alpha_{k} \circ h_{k} \circ p_{k}^{k+1}(z)=s_{k+1}(z)$ for each $z \in\left(p_{k}^{k+1}\right)^{-1}\left(h_{k}^{-1}\left(\left|N_{k}\right|\right)\right)$.

Now we apply Lemma 4.1 with $\left(X, Z, U, C, p, Z_{0}, \Gamma^{*}, \Gamma, \mathcal{U}, \mathcal{V}, g, N_{0}\right)$ replaced by

$$
\left(X_{i}, \operatorname{cl}_{X_{i}}\left(S_{i}\right), W_{i}, S_{i+1}, p_{i}^{i+1} \mid S_{i+1}, Z_{i}, \Gamma, \Gamma, \mathcal{W}_{i}, \mathcal{W}_{i+1}, s_{i}, N_{i}\right) .
$$

We ask the reader to make the routine check that all the hypotheses of Lemma 4.1 are satisfied with this input.

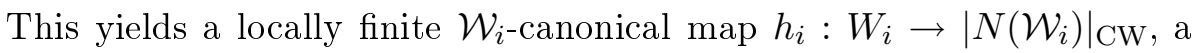
locally finite $\mathcal{W}_{i+1}$-canonical map $s_{i+1}: W_{i+1} \rightarrow\left|N\left(\mathcal{W}_{i+1}\right)\right|_{\mathrm{CW}}$ along with the minimal subcomplex $N_{i+1}$ of $N\left(\mathcal{W}_{i+1}\right)$ such that $s_{i+1}\left(Z_{i+1}\right) \subset\left|N_{i+1}\right|$, and a simplicial injection $\alpha_{i}: N_{i} \rightarrow N\left(\mathcal{W}_{i+1}\right), \alpha_{i}\left(W_{i, v}\right)=W_{i+1, v}$, subject to certain properties. Indeed, it is clear that all of (I1)-(I3) are satisfied except perhaps that we need to check $\alpha_{i}\left(N_{i}\right) \subset N_{i+1}$. But we have established from (F4) that $Z_{i} \subset p_{i}^{i+1}\left(Z_{i+1}\right)$. This, the ultimate statement of Lemma 4.1, and an application of Lemma 3.7 of [7] show that if $\left\{W_{i, v} \mid v \in \Gamma_{0}\right\}$ is the set of vertices of a simplex of $N_{i}$, then $\left\{\alpha_{i}\left(W_{i, v}\right) \mid v \in \Gamma_{0}\right\}=\left\{W_{i+1, v} \mid v \in \Gamma_{0}\right\}$ is the set of vertices of a simplex of $N_{i+1}$. Our inductive construction is complete.

For each $i \in \mathbb{N}$, define $M_{i}=\operatorname{int}_{X_{i}} h_{i}^{-1}\left(\left|N_{i}\right|\right)$ and set $\mathbf{M}=\left(M_{i}\right)$. Then $\mathbf{M}$ is an open semi-sequence of $\mathbf{X}$; to see that $\mathbf{M}$ is expanding, let $i \in \mathbb{N}$ and $t \in M_{i}$. By (I3), if $z \in\left(p_{i}^{i+1}\right)^{-1}(t)$, then $s_{i+1}(z) \in\left|N_{i+1}\right|$. Thus $z \in s_{i+1}^{-1}\left(\left|N_{i+1}\right|\right)$; from (I2) it follows that $z \in \operatorname{int}_{X_{i+1}} h_{i+1}^{-1}\left(\left|N_{i+1}\right|\right)=M_{i+1}$.

Clearly $M_{i} \subset W_{i}$ for each $i \in \mathbb{N}$, so $\operatorname{slim} \mathbf{M} \subset \operatorname{sim} \mathbf{W}=W$. To show the opposite inclusion, suppose that $x \in W=\operatorname{slim} \mathbf{S}$. Put $k=\Phi_{\mathbf{S}}(x)$, the S-birth index of $x$. Hence $p_{i}(x) \in p_{i}(W) \cap S_{i} \subset Z_{i}$ for all $i \geq k$. Using (I1) and (I2), one sees that $p_{i}(x) \in M_{i}$ for all $i \geq k$, which implies that $x \in \operatorname{sim} \mathbf{M}$. 
We now see that (1) of our lemma has been verified, so we proceed with (2). By dint of (F3), for each $i \in \mathbb{N}$, there is a simplicial injection $\beta_{i}: N_{i} \rightarrow N(\mathcal{W})$ obtained from the vertex map $W_{i, v} \mapsto W_{v}$. Hence we get a map $g_{i}: M_{i} \rightarrow|K|_{\text {CW }}$ defined by $g_{i}(x)=\kappa \circ \beta_{i} \circ h_{i}(x)$ for all $x \in M_{i}$.

If $x \in M_{i+1}$ and $p_{i}^{i+1}(x) \in M_{i}$, then $h_{i} \circ p_{i}^{i+1}(x) \in h_{i}\left(M_{i}\right) \subset\left|N_{i}\right|$. From (I3), $s_{i+1}(x)=\alpha_{i} \circ h_{i} \circ p_{i}^{i+1}(x) \in\left|N_{i+1}\right|$. Using (I2), we find that $h_{i+1}(x)=$ $\alpha_{i} \circ h_{i} \circ p_{i}^{i+1}(x)$, which implies that $\beta_{i+1} \circ h_{i+1}(x)=\beta_{i+1} \circ \alpha_{i} \circ h_{i} \circ p_{i}^{i+1}(x)$. But $\beta_{i+1} \circ \alpha_{i}=\beta_{i}$ as one can easily check. Therefore $\kappa \circ \beta_{i+1} \circ h_{i+1}(x)=$ $\kappa \circ \beta_{i} \circ h_{i} \circ p_{i}^{i+1}(x)$, i.e., $g_{i+1}(x)=g_{i} \circ p_{i}^{i+1}(x)$. This proves that $\mathbf{g}$ is a map of $\mathbf{M}$ to $|K|_{\mathrm{CW}}$. Let $g=\operatorname{slim} \mathbf{g}: W \rightarrow|K|_{\mathrm{CW}}$.

To obtain the $K$-modification part, let $x \in W, i=\Phi_{\mathbf{M}}(x)$, and $z=p_{i}(x)$. Then $g(x)=g_{i} \circ p_{i}(x)=g_{i}(z)=\kappa \circ \beta_{i} \circ h_{i}(z)$, and $h_{i}(z) \in\left|N_{i}\right|$; suppose that $W_{i, v}$ is a vertex of the simplex of $N_{i}$ that contains $h_{i}(z)$ in its interior. Now $x \in p_{i}^{-1}\left(W_{i, v}\right) \subset W_{v}=f^{-1}(\operatorname{st}(v, K))$. This shows that $v$ is in $\sigma$ where $\sigma$ is the simplex of $K$ such that $f(x) \in \operatorname{int} \sigma$. We know that $\kappa \circ \beta_{i}\left(W_{i, v}\right)=v$, so $g(x) \in \sigma$. Our proof is complete.

Lemma 4.4. Let $\mathbf{X}=\left(X_{i}, p_{i}^{i+1}\right)$ be an inverse sequence of stratifiable spaces, $K$ a CW-complex, $W$ an open subset of $X=\lim \mathbf{X}$, and $f: W \rightarrow K$ a map. Then there exist:

(1) an expanding open semi-sequence $\mathbf{M}=\left(M_{i}\right)$ of $\mathbf{X}$ with $\operatorname{sim} \mathbf{M}=W$,

(2) a map $\mathbf{g}$ of $\mathbf{M}$ to $K$ such that the map $g=\operatorname{slim} \mathbf{g}: W \rightarrow K$ is homotopic to $f$.

Proof. Select a simplicial complex $L$ having the property that $|L|_{\mathrm{CW}}$ is homotopy equivalent to $K$, and let $\alpha: K \rightarrow|L|_{\mathrm{CW}}$ and $\beta:|L|_{\mathrm{CW}} \rightarrow K$ be a homotopy equivalence and a homotopy inverse, respectively. Let $f_{0}=\alpha \circ f$ : $W \rightarrow|L|_{\mathrm{CW}}$.

Apply Lemma 4.3 to $f_{0}$ and obtain $\mathbf{M}$ and a map $\mathbf{g}_{0}$ of $\mathbf{M}$ to $|L|_{\mathrm{CW}}$ as in (1) and (2) of that lemma. Of course $\mathbf{M}$ meets the requirements of (1) of the current lemma, so we have to get (2).

Put $\mathbf{g}=\beta \circ \mathbf{g}_{0}$; then $\mathbf{g}$ is a map of $\mathbf{M}$ to $K$. We know that $g_{0}=\operatorname{slim} \mathbf{g}_{0}$ is an $L$-modification of $f_{0}$. Consequently, $g_{0} \simeq f_{0}$, so $\beta \circ g_{0} \simeq \beta \circ f_{0}=$ $\beta \circ \alpha \circ f \simeq f$, and $g=\operatorname{sim} \mathbf{g}=\beta \circ \operatorname{slim} \mathbf{g}_{0}=\beta \circ g_{0} \simeq f$, as required by (2).

\section{Proofs of characterization theorems}

(i) Proof of $(\Leftarrow)$ of Theorems 3.1 and 3.2. Let $A$ be closed in $X$ and $f: A \rightarrow|K|_{\mathrm{CW}}$ (or $K$ ) be a map. We may assume that $f$ is defined on an open neighborhood $W$ of $A$. Apply Lemma 4.3 to the map $f: W \rightarrow|K|_{\mathrm{CW}}$ to obtain an expanding open semi-sequence $\mathbf{M}$ of $\mathbf{X}, W=\operatorname{sim} \mathbf{M}$, a map g : $\mathbf{M} \rightarrow|K|_{\mathrm{CW}}$, and $g=\operatorname{slim} \mathbf{g}: W \rightarrow|K|_{\mathrm{CW}}$. The map $g$ is a $K$ modification of $f$; consequently, $g$ is contiguous to $f$ and $g|A \simeq f| A$. (In the 
CW case, apply Lemma 4.4 instead of Lemma 4.3; the above homotopy is still true.)

Put $H=X \backslash A$ and let $\mathbf{H}$ be the semi-sequence of $\mathbf{X}$ induced by $\{H\}$. Then $H=\operatorname{sim} \mathbf{H}$ and $\mathbf{H}$ is open and expanding in $\mathbf{X}$. Let us also note that $X=W \cup H$.

All the conditions of the sufficiency of Theorem 3.2 (or 3.1) have been satisfied by the preceding data. Therefore we may now assume the existence of expanding open subsemi-sequences $\mathbf{M}^{*}$ of $\mathbf{M}, \mathbf{H}^{*}$ of $\mathbf{H}$, and a map $\mathbf{g}^{*}$ : $\mathbf{M}^{*} \cup \mathbf{H}^{*} \rightarrow|K|_{\mathrm{CW}}\left(\right.$ or $K$ ) such that $\operatorname{sim} \mathbf{M}^{*} \cup \operatorname{sim} \mathbf{H}^{*}=\operatorname{slim} \mathbf{M} \cup \operatorname{slim} \mathbf{H}=$ $W \cup H=X$ and $\mathbf{g}_{0}^{*}=\mathbf{g}^{*} \mid \mathbf{M}^{*}$ is a $K$-modification of (homotopic to) $\mathbf{g}_{0}=$ $\mathbf{g} \mid \mathbf{M}^{*}$, and in either version $\mathbf{g}_{0}^{*} \simeq \mathbf{g}_{0}$.

Note that $\mathbf{M}^{*}, \mathbf{H}^{*}$ are expanding open; therefore $\operatorname{sim}\left(\mathbf{M}^{*} \cup \mathbf{H}^{*}\right)=$ $\operatorname{sim} \mathbf{M}^{*} \cup \operatorname{sim} \mathbf{H}^{*}=X$.

By Lemma $2.4, g^{*}=\operatorname{slim} \mathbf{g}^{*}: X \rightarrow|K|_{\mathrm{CW}}($ or $K$ ) is a map.

Let $D=\operatorname{sim} \mathbf{M}^{*}, g_{0}^{*}=\operatorname{slim} \mathbf{g}_{0}^{*}: D \rightarrow|K|_{\mathrm{CW}}\left(\right.$ or to $K$ ) and $g_{0}=\operatorname{sim} \mathbf{g}_{0}$ : $D \rightarrow|K|_{\mathrm{CW}}\left(\right.$ or to $K$ ). The homotopy $\mathbf{g}_{0}^{*} \simeq \mathbf{g}_{0}$ and Lemma 2.10 show that $g_{0}^{*}$ and $g_{0}$ are maps, and $g_{0}^{*} \simeq g_{0}$. Note that $g_{0}^{*}=g^{*} \mid D$ and $g_{0}|D=g| D$. Hence, $g^{*}|D \simeq g| D$.

Since $A \cap H=\emptyset$, we have $A \subset D=\operatorname{sim} \mathbf{M}^{*}$. From this and the preceding, it follows that $g^{*}|A \simeq g| A$. We have already established that $g|A \simeq f| A$. Since $g^{*}: X \rightarrow|K|_{\mathrm{CW}}$ (or $K$ ), our proof of $(\Leftarrow)$ is completed by applying the homotopy extension theorem.

Let us point out that $(\Rightarrow)$ of Theorem 3.2 is used in the proof of $(\Rightarrow)$ of Theorem 3.1, so we separate the two proofs.

(ii) Proof of $(\Rightarrow)$ of Theorem 3.2. Suppose that $\mathbf{M}=\left(M_{i}\right), \mathbf{H}=\left(H_{i}\right)$ are expanding open semi-sequences of $\mathbf{X}$, and $\mathbf{g}=\left(g_{i}\right)$ is a map of $\mathbf{M}$ to $|K|_{\mathrm{CW}}$. Let $M=\operatorname{slim} \mathbf{M}$ and $H=\operatorname{sim} \mathbf{H}$. Lemma 2.4 shows that $M$ and $H$ are open in $X$ and that $g=\operatorname{sim} \mathbf{g}: M \rightarrow|K|_{\mathrm{CW}}$ is a map.

Consider a surjective extension $\widetilde{\mathbf{X}}$ of $\mathbf{X}$ as in Lemma 2.8. We shall determine a particular expanding open semi-sequence $\widetilde{\mathbf{M}}=\left(\widetilde{M}_{i}\right)$ of $\widetilde{\mathbf{X}}$ which is an extension of $\mathbf{M}$. Let $\widetilde{M}_{1}=M_{1}$. Suppose that $k \in \mathbb{N}$ and we have determined $\widetilde{M}_{i}$ for $1 \leq i \leq k$ in such a manner that always:

(M1) $\quad \widetilde{M}_{i} \cap X_{i}=M_{i}$.

We require, moreover, that if $1 \leq i<k$ then:

(M2) $\left(\widetilde{p}_{i}^{i+1}\right)^{-1}\left(\widetilde{M}_{i}\right) \subset \widetilde{M}_{i+1}$,

(M3) if $x \in \widetilde{M}_{i}$, then for some $y \in \widetilde{M}_{i+1}, \widetilde{p}_{i}^{i+1}(y)=x$,

(M4) if $x \in \widetilde{M}_{i+1} \backslash M_{i+1}$, then $\widetilde{p}_{i}^{i+1}(x) \in \widetilde{M}_{i}$.

One selects $\widetilde{M}_{k+1}$ by adjoining to $M_{k+1}$ the minimum set of points in $D_{k+1}$ necessary to make (M3) true in case $i$ is replaced by $k$. This way the 
expanding open semi-sequence $\mathbf{M} \subset \mathbf{X}$ "extends" to an expanding open semi-sequence $\widetilde{\mathbf{M}} \subset \widetilde{\mathbf{X}}$, and by Lemma $2.7, \operatorname{sim}_{\widetilde{\mathbf{X}}} \widetilde{\mathbf{M}} \cap X=\operatorname{sim}_{\mathbf{X}}(\widetilde{\mathbf{M}} \cap \mathbf{X})$ $=M$. Let $\widetilde{\mathbf{H}}$ be an analogous "extension" of $\mathbf{H}$. Define $\widetilde{M}=\operatorname{sim}_{\widetilde{\mathbf{X}}} \widetilde{\mathbf{M}}$ and $\widetilde{H}=\operatorname{sim}_{\widetilde{\mathbf{x}}} \widetilde{\mathbf{H}}$. Then $\widetilde{M} \cap X=M, \widetilde{H} \cap X=H$.

Extend $\mathbf{g}$ to $\widetilde{\mathbf{g}}: \widetilde{\mathbf{M}} \rightarrow|K|_{\mathrm{CW}}$ inductively, by defining $\widetilde{g}_{1}=g_{1}, \widetilde{g}_{i+1} \mid M_{i+1}$ $=g_{i+1}$, and, using $(\mathrm{M} 4), \widetilde{g}_{i+1}(x)=\widetilde{g}_{i} \circ \widetilde{p}_{i}^{i+1}(x)$ whenever $x \in \widetilde{M}_{i+1} \backslash M_{i+1}$.

Note that $\widetilde{M} \cup \widetilde{H}$ is a normal space. An application of the shrinking theorem shows that there is an open subset $\widetilde{Q}$ of $\widetilde{M} \cup \widetilde{H}$ whose closure $Q_{0}$ with respect to $\widetilde{M} \cup \widetilde{H}$ is a subset of $\widetilde{M}$ and such that $\widetilde{Q} \cup \widetilde{H}=\widetilde{M} \cup \widetilde{H}$. Observe that $(\widetilde{Q} \cup \widetilde{H}) \cap X=M \cup H$. Since $\widetilde{M} \cup \widetilde{H}$ is an absolute co-extensor for $|K|_{\mathrm{CW}}$ (Theorem 3.6 of [6]), choose a map $\widetilde{f}: \widetilde{M} \cup \widetilde{H} \rightarrow|K|_{\mathrm{CW}}$ having the property that $\widetilde{f}\left|Q_{0}=\widetilde{g}\right| Q_{0}: Q_{0} \rightarrow|K|_{\mathrm{CW}}$.

Let us apply Lemma 4.3 to the map $\widetilde{f}$ and the open subset $\widetilde{M} \cup \widetilde{H}$ of $\widetilde{X}$. We obtain an expanding open semi-sequence $\widetilde{\mathbf{N}}=\left(\widetilde{N}_{i}\right)$ of $\widetilde{\mathbf{X}}$ and a map $\widetilde{\mathbf{h}}=\left(\widetilde{h}_{i}\right)$ of $\widetilde{\mathbf{N}}$ to $|K|_{\mathrm{CW}}$. One sees that $\operatorname{sim} \widetilde{\mathbf{N}}=\widetilde{M} \cup \widetilde{H}$; hence the map $\widetilde{h}=\operatorname{sim} \widetilde{\mathbf{h}}: \widetilde{M} \cup \widetilde{H} \rightarrow|K|_{\mathrm{CW}}$ is a $K$-modification of $\widetilde{f}$.

Find an expanding open semi-sequence $\widetilde{\mathbf{Q}}=\left(\widetilde{Q}_{i}\right)$ of $\widetilde{\mathbf{X}}$ such that $\operatorname{sim} \widetilde{\mathbf{Q}}$ $=\widetilde{Q}$. Define $\widetilde{\mathbf{M}}^{*}=\widetilde{\mathbf{M}} \cap \widetilde{\mathbf{N}} \cap \widetilde{\mathbf{Q}}$. Then $\widetilde{\mathbf{M}}^{*}$ is a subsemi-sequence of $\widetilde{\mathbf{M}}$; similarly $\widetilde{\mathbf{H}}^{*}=\widetilde{\mathbf{H}} \cap \widetilde{\mathbf{N}}$ is a subsemi-sequence of $\widetilde{\mathbf{H}}$. Note that $\widetilde{\mathbf{M}}^{*}, \widetilde{\mathbf{H}}^{*}$ are expanding open semi-sequences of $\widetilde{\mathbf{X}}$.

Observe that $\widetilde{Q}=\operatorname{slim} \widetilde{\mathbf{Q}} \subset \widetilde{M}=\operatorname{sim} \widetilde{\mathbf{M}} \subset \widetilde{M} \cup \widetilde{H}=\operatorname{slim} \widetilde{\mathbf{N}}$. Since $\widetilde{\mathbf{M}}, \widetilde{\mathbf{N}}$ are expanding, $\widetilde{\mathbf{M}} \cap \widetilde{\mathbf{N}}$ is expanding and $\operatorname{sim}(\widetilde{\mathbf{M}} \cap \widetilde{\mathbf{N}})=\operatorname{sim} \widetilde{\mathbf{M}} \cap \operatorname{slim} \widetilde{\mathbf{N}}=$ $\widetilde{M} \cap(\widetilde{M} \cup \widetilde{H})=\widetilde{M}=\operatorname{slim} \widetilde{\mathbf{M}}$. Since $\widetilde{\mathbf{Q}}$ is expanding, we may apply the preceding reasoning again to see that $\operatorname{sim} \widetilde{\mathbf{M}}^{*}=\operatorname{sim}(\widetilde{\mathbf{M}} \cap \widetilde{\mathbf{N}} \cap \widetilde{\mathbf{Q}})=\operatorname{sim} \widetilde{\mathbf{Q}}$ $=\widetilde{Q}$. In a similar manner, one sees that $\operatorname{sim} \widetilde{\mathbf{H}}^{*}=\operatorname{sim} \widetilde{\mathbf{H}} \cap \operatorname{sim} \widetilde{\mathbf{N}}=\widetilde{H} \cap$ $(\widetilde{M} \cup \widetilde{H})=\widetilde{H}$. Hence $\operatorname{sim} \widetilde{\mathbf{M}}^{*} \cup \operatorname{sim} \widetilde{\mathbf{H}}^{*}=\widetilde{Q} \cup \widetilde{H}=\widetilde{M} \cup \widetilde{H}=\operatorname{sim} \widetilde{\mathbf{M}} \cup \operatorname{sim} \widetilde{\mathbf{H}}$.

Now, take $\mathbf{M}^{*}=\widetilde{\mathbf{M}}^{*} \cap \mathbf{X}$ and $\mathbf{H}^{*}=\widetilde{\mathbf{H}}^{*} \cap \mathbf{X}$ which are expanding open semi-sequences of $\mathbf{X}$. Also $\mathbf{M}^{*} \subset \mathbf{M}, \mathbf{H}^{*} \subset \mathbf{H}$ and $\operatorname{sim} \mathbf{M}^{*} \cup \operatorname{sim} \mathbf{H}^{*}=$ $\operatorname{sim}\left(\widetilde{\mathbf{M}}^{*} \cap \mathbf{X}\right) \cup \operatorname{slim}\left(\widetilde{\mathbf{H}}^{*} \cap \mathbf{X}\right)=M \cup H$. This gives us (1) of Theorem 3.2.

As $\widetilde{\mathbf{M}}^{*} \cup \widetilde{\mathbf{H}}^{*}$ is a subsemi-sequence of $\widetilde{\mathbf{N}}$, we may define $\widetilde{\mathbf{g}}^{*}=\widetilde{\mathbf{h}} \mid \widetilde{\mathbf{M}}^{*} \cup \widetilde{\mathbf{H}}^{*}$ : $\widetilde{\mathbf{M}}^{*} \cup \widetilde{\mathbf{H}}^{*} \rightarrow|K|_{\mathrm{CW}}$. Write $\widetilde{\mathbf{g}}^{*}=\left(\widetilde{g}_{i}^{*}\right)$. Let us prove that $\widetilde{\mathbf{g}}^{*} \mid \widetilde{\mathbf{M}}^{*}$ is a $K$ modification of $\widetilde{\mathbf{g}} \mid \widetilde{\mathbf{M}}^{*}$.

Fix $i \in \mathbb{N}$ and suppose that $z \in \widetilde{M}_{i} \cap \widetilde{N}_{i} \cap \widetilde{Q}_{i} \subset \widetilde{Q}_{i}$. The fact that $\widetilde{\mathbf{Q}}$ is expanding implies that $\widetilde{p}_{i}^{-1}\left(\widetilde{Q}_{i}\right) \subset \widetilde{Q}$. Since the bonding maps in $\widetilde{\mathbf{X}}$ are surjective and $\widetilde{\mathbf{Q}}$ is expanding, there exists $x \in \widetilde{p}_{i}^{-1}\left(\widetilde{Q}_{i}\right) \subset \widetilde{Q} \subset Q_{0} \subset \widetilde{M}=$ $\operatorname{sim} \widetilde{\mathbf{M}}$ with $\widetilde{p}_{i}(x)=z$. According to Lemma 2.4, $\widetilde{g}(x)=\widetilde{g}_{i} \circ \widetilde{p}_{i}(x)=\widetilde{g}_{i}(z)$.

Let $\sigma$ be the simplex of $K$ such that $\widetilde{f}(x)=\widetilde{g}(x)=\widetilde{g}_{i}(z) \in \operatorname{int} \sigma$. We know that $\widetilde{h}$ is a $K$-modification of $\widetilde{f}$, so $\widetilde{h}(x) \in \sigma$. Notice that $\widetilde{h}(x)=\widetilde{h}_{i} \circ \widetilde{p}_{i}(x)=$ 
$\widetilde{g}_{i}^{*} \circ \widetilde{p}_{i}(x)=\widetilde{g}_{i}^{*}(z)$. Hence $\widetilde{g}_{i}^{*}(z) \in \sigma$. Therefore $\widetilde{g}_{i}^{*}$ is a $K$-modification of $\widetilde{g}_{i}$. This shows that $\widetilde{\mathbf{g}}^{*} \mid \widetilde{\mathbf{M}}^{*}$ is a $K$-modification of $\widetilde{\mathbf{g}} \mid \widetilde{\mathbf{M}}^{*}$.

Finally, put $\mathbf{g}^{*}=\widetilde{\mathbf{g}}^{*} \mid\left(\mathbf{M}^{*} \cup \mathbf{H}^{*}\right)$. Since the restriction of a $K$-modification is a $K$-modification we conclude that $\mathbf{g}^{*} \mid \mathbf{M}^{*}$ is a $K$-modification of $\widetilde{\mathbf{g}} \mid \mathbf{M}^{*}=$ $\mathrm{g} \mid \mathbf{M}^{*}$.

(iii) Proof of $(\Rightarrow)$ of Theorem 3.1. Suppose that $\mathbf{M}, \mathbf{H}$ are expanding open semi-sequences of $\mathbf{X}$ and $\mathbf{g}$ is a map of $\mathbf{M}$ to $K$. Find a simplicial complex $L$ along with a homotopy equivalence $\alpha: K \rightarrow|L|_{\mathrm{CW}}$ and a homotopy inverse $\beta:|L|_{\mathrm{CW}} \rightarrow K$ of $\alpha$.

Now $X$ is an absolute co-extensor for $|L|_{\mathrm{CW}}$, so we apply (ii) to the map $\alpha \circ \mathbf{g}$ from $\mathbf{M}$ to $|L|_{\mathrm{CW}}$. In this case, the map $\mathbf{g}^{*} \mid \mathbf{M}^{*}$ is an $L$-modification of $(\alpha \circ \mathbf{g}) \mid \mathbf{M}^{*}$; by Lemma 2.13 these maps are homotopic. Then $\beta \circ \mathbf{g}^{*} \mid \mathbf{M}^{*} \simeq$ $\beta \circ\left(\alpha \circ \mathbf{g} \mid \mathbf{M}^{*}\right)$ and the fact that $\alpha$ and $\beta$ are homotopy inverses imply that $\beta \circ \mathbf{g}^{*}\left|\mathbf{M}^{*} \simeq \mathbf{g}\right| \mathbf{M}^{*}$.

\section{References}

[1] C. Borges, On stratifiable spaces, Pacific J. Math. 17 (1966), 1-16.

[2] R. Cauty, Convexité topologique et prolongement des fonctions continues, Compos. Math. 27 (1973), 233-273.

[3] J. Ceder, Some generalizations of metric spaces, Pacific J. Math. 11 (1961), 105-125.

[4] A. Chigogidze and V. Valov, Universal metric spaces and extension dimension, Topology Appl. 113 (2001), 23-27.

[5] A. Dranishnikov and J. Dydak, Extension dimension and extension types, Trudy Mat. Inst. Steklova 212 (1996), 61-94 (in Russian).

[6] I. Ivanšić and L. Rubin, The extension dimension of universal spaces, Glas. Mat. Ser. III 38(58) (2003), 121-127.

[7] - - - Limit theorem for semi-sequences in extension theory, Houston J. Math. 31 (2005), 787-807.

[8] - - - Some applications of semi-sequences to extension theory, Topology Proc., to appear.

[9] M. Levin, On extensional dimension of metrizable spaces, preprint.

[10] S. Mardešić, Extension dimension of inverse limits, Glas. Mat. Ser. III 35(55) (2000), 339-354.

[11] S. Mardešić and J. Segal, Shape Theory, North-Holland, Amsterdam, 1982.

[12] R. Millspaugh, L. Rubin and P. Schapiro, Irreducible representations of metrizable spaces, and strongly countable-dimensional spaces, Fund. Math. 148 (1995), 223-256.

[13] K. Nagami, Finite-to-one closed mappings and dimension. II, Proc. Japan Acad. 35 (1959), 437-439.

[14] -, Mappings of finite order, Japan J. Math. 30 (1960), 25-54.

[15] L. Rubin, Cohomological dimension and approximate limits, Proc. Amer. Math. Soc. 125 (1997), 3125-3128.

[16] -, A stronger limit theorem in extension theory, Glas. Mat. Ser. III 36(56) (2001), 95-103. 
[17] L. Rubin and P. Schapiro, Limit theorem for inverse sequences of metric spaces in extension theory, Pacific J. Math. 187 (1999), 177-186.

Ivan Ivanšić

Department of Mathematics

University of Zagreb

Unska 3

P.O. Box 148, 10001 Zagreb, Croatia

E-mail: ivan.ivansic@fer.hr
Leonard R. Rubin Department of Mathematics University of Oklahoma Norman, OK 73019, U.S.A.

E-mail: lrubin@ou.edu 